\title{
DESENVOLVIMENTO DE UM DISPOSITIVO DE BAIXO CUSTO PARA MEDIDAS POR QUIMILUMINESCÊNCIA
}

\author{
Eduardo P. Borges, Elizabeth N. Fernandes e Fábio R. P. Rocha ${ }^{\#}$ \\ Departamento de Química, Universidade Federal de São Carlos, CP 676, 13565-905 São Carlos - SP \\ Boaventura F. Reis* \\ Centro de Energia Nuclear na Agricultura, Universidade de São Paulo, CP 96, 13400-970 Piracicaba - SP
}

Recebido em 29/11/01; aceito em 27/5/02

\begin{abstract}
DEVELOPMENT OF A LOW COST DEVICE FOR CHEMILUMINESCENCE MEASUREMENTS. A simple and low cost device (ca. US\$ 150) that comprises two photodiodes fixed in lab-made Perspex flow cell is proposed for chemiluminescence measurements. The characteristics of the device (large observation window and reduced thickness) allow maximizing the amount of the emitted radiation detected. A sensitivity improvement of ca. $50 \%$ was observed by employing two photodiodes for signal measurements. The performance of the device was assessed by the oxidation of luminol by hydrogen peroxide, yielding a linear

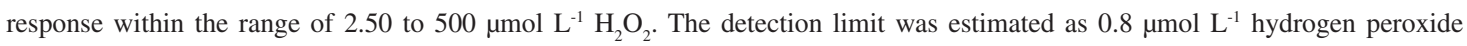
which is comparable with those obtained by using equipments based on photomultipliers.
\end{abstract}

Keywords: flow analysis; chemiluminescence; hydrogen peroxide; photodiode.

\section{INTRODUÇÃO}

Procedimentos analíticos com detecção por quimiluminescência destacam-se pela alta sensibilidade e ampla faixa de resposta linear ${ }^{1}$. Tais métodos podem ser implementados empregando instrumentação similar à utilizada em outros métodos ópticos, como espectrofotometria e fluorescência. Entretanto, os equipamentos são usualmente mais simples devido à ausência de fontes de radiação externas e de dispositivos para a discriminação de comprimentos de onda ${ }^{2}$.

Quimiluminescência é, geralmente, monitorada empregando celas em espiral posicionadas em frente à janela de uma fotomultiplicadora ${ }^{3}$. Apesar de procedimentos bastante sensíveis serem implementados com estes dispositivos, sua aplicação é limitada ao uso em laboratório devido à fragilidade. $\mathrm{O}$ emprego de quimiluminescência para medidas em campo, por exemplo, requer o desenvolvimento de dispositivos mais robustos e versáteis. Adicionalmente, apesar da emissão de radiação ocorrer em todas as direções, com as configurações dos equipamentos usuais, apenas uma fração da radiação é detectada devido ao posicionamento da cela de medida em relação ao detector.

Os fotodiodos, embora menos sensíveis que as fotomultiplicadoras, podem ser usados para a detecção da radiação emitida, apresentando vantagens como maior robustez e menores custo e tamanho. O emprego destes dispositivos é particularmente interessante no caso de sistemas miniaturizados ou destinados a medidas em campo ${ }^{4}$. Devido aos menores custos e tamanho, mais de um fotodiodo pode ser empregado para maximizar a quantidade de radiação detectada.

Neste trabalho, é proposto um dispositivo simples, robusto e de baixo custo para medidas por quimiluminescência em sistemas de análise em fluxo. Visando melhorar a sensibilidade, foi investigado o emprego de dois fotodiodos acoplados à cela de fluxo. O desempenho do dispositivo foi avaliado empregando como modelo a oxidação de luminol por peróxido de hidrogênio em meio alcalino.

*e-mail: reis@cena.usp.br

\# endereço atual: Instituto de Química, Universidade de São Paulo, CP 26077. 05513-970 São Paulo - SP

\section{PARTE EXPERIMENTAL}

\section{Descrição do dispositivo}

O dispositivo proposto é mostrado na Figura 1 e consiste de uma cela de fluxo similar a anteriormente proposta para medidas por espectrofotometria em fase sólida ${ }^{5}$, à qual foram acoplados dois fotodiodos de silício (a, b) com área sensível de $50 \mathrm{~mm}^{2}$ (OSD50-E, RS 846-711). A cela de fluxo foi construída empregando peças de acrílico e uma tira de borracha com $1 \mathrm{~mm}$ de espessura (c), presas por parafusos (não mostrados na figura). Na parte central da tira de borracha, foi feito um corte circular com $10 \mathrm{~mm}$ de diâmetro (área transversal de $\left.78 \mathrm{~mm}^{2}\right)$, sendo o volume da cela $(78 \mu \mathrm{L})$ definido pela a espessura da tira e pela área do corte. Essa área também correspondia à janela de observação, que permite que a radiação emitida pela reação alcance os fotodetectores. Nos dois blocos do lado direito da figura, foram feitos dois furos transversais $(0,8 \mathrm{~mm}$ d.i.), distantes $30 \mathrm{~mm}$, para a entrada e saída de fluidos. Na tira de borracha, foi feito um corte para interligar os pontos de entrada e saída de fluidos à área de observação. O conjunto foi acondicionado em uma caixa escura para evitar a incidência de luz ambiente sobre os fotodetectores.

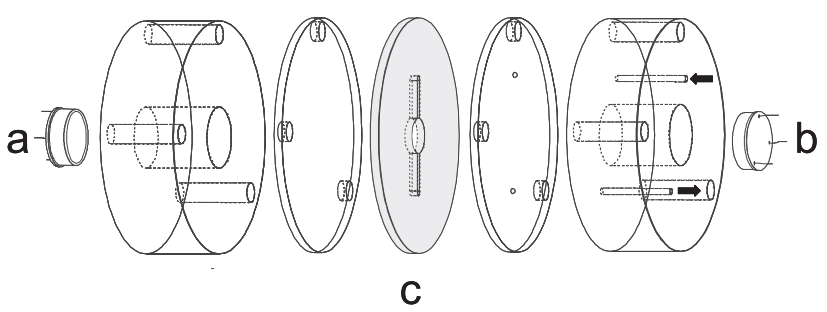

Figura 1. Dispositivo empregado para medidas por quimiluminescência. $a$, $b$-fotodiodos de silício com $50 \mathrm{~mm}^{2}$ de área sensível; c - tira de borracha com $1 \mathrm{~mm}$ de espessura. As demais peças foram construídas em acrílico. As setas indicam os pontos de entrada e saída de soluções 
O diagrama do circuito eletrônico empregado para o condicionamento dos sinais gerados pelos fotodiodos é mostrado na Figura 2. Os terminais de saída de cada fotodiodo foram conectados às entradas não inversoras de dois amplificadores operacionais (CA3140) para amplificação e compatibilização de impedância. Os terminais de saída destes dispositivos foram conectados a um amplificador configurado como somador de sinais, que também permitia o ajuste de zero ("offset"). O sinal de saída do amplificador foi monitorado empregando um registrador potenciométrico ou através de um microcomputador, equipado com uma interface eletrônica. Uma fonte de diferença de potencial constante bipolar $( \pm 12 \mathrm{~V})$ foi empregada para a alimentação dos dispositivos.

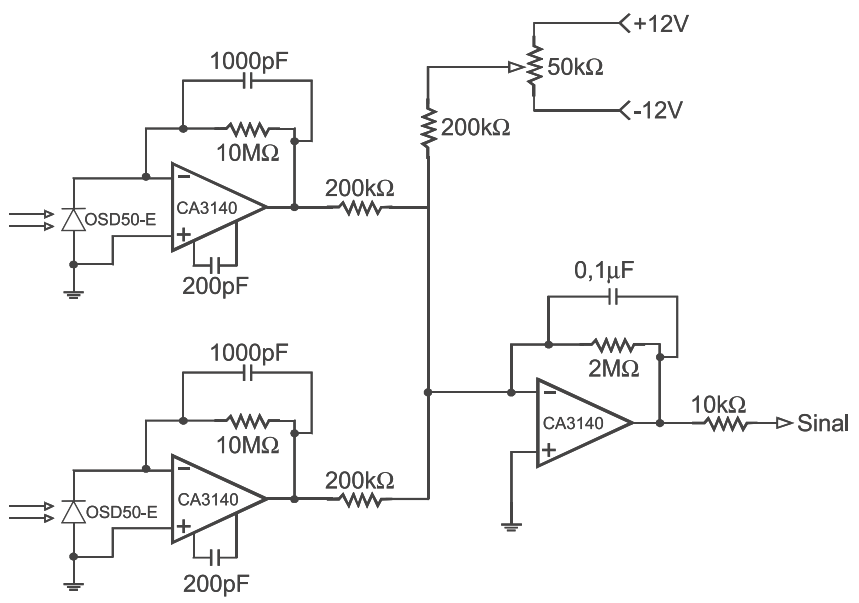

Figura 2. Circuito eletrônico empregado para o condicionamento dos sinais e ajuste de zero ("offset"). A saída de sinal foi conectada a um registrador potenciométrico ou a uma das entradas analógicas da interface PCL-711S

\section{Reagentes e soluções}

Todas as soluções foram preparadas empregando água deionizada e reagentes com grau analítico. Solução $4,50 \mathrm{mmol} \mathrm{L}{ }^{-1}$ luminol $\left(\mathrm{R}_{1}\right)$ foi preparada dissolvendo-se 79,6 mg de 5-amino-2,3-diidro-1,4 ftalazinediona (Sigma) em $100 \mathrm{~mL}$ de solução $0,2 \mathrm{~mol} \mathrm{~L}^{-1} \mathrm{~K}_{2} \mathrm{CO}_{3} \mathrm{e}$ ajustando o pH para $10,5 \mathrm{com} \mathrm{HCl}$. Solução $50,0 \mathrm{mmol} \mathrm{L}^{-1}$ hexacianoferrato(III) de potássio $\left(\mathrm{R}_{2}\right)$ foi preparada dissolvendo-se $1,65 \mathrm{~g}$ de $\mathrm{K}_{3} \mathrm{Fe}(\mathrm{CN})_{6}$ em $100 \mathrm{~mL}$ de água.

Solução estoque de peróxido de hidrogênio (ca. $1 \mathrm{mmol} \mathrm{L}^{-1}$ ) foi preparada a partir de uma solução $30 \% \mathrm{~m} / \mathrm{m}$ e padronizada com permanganato de potássio. Soluções de referência foram preparadas por diluições da solução estoque.

\section{Equipamentos}

O módulo de análise foi construído empregando tubos de polietileno $(0,8 \mathrm{~mm}$ d.i.) e confluências feitas de acrílico. A propulsão dos fluidos foi efetuada por uma bomba peristáltica Ismatec IPC-
4, equipada com tubos de propulsão de Tygon. Para a inserção das soluções foi empregado um injetor proporcional com barra deslizante. Um registrador potenciométrico (Cole-Parmer) ou um microcomputador equipado com uma interface eletrônica (Advantech, PCL711S) foram empregados para o monitoramento dos sinais.

\section{Diagrama de fluxos e procedimento}

O instrumento foi avaliado empregando a reação de oxidação de luminol por peróxido de hidrogênio, catalisada por hexacianoferrato(III) de potássio. O diagrama de fluxos do módulo de análise empregado é mostrado na Figura 3. As soluções de peróxido de hidrogênio $(100 \mu \mathrm{L})$ foram inseridas no fluido transportador, recebendo por confluência as soluções de luminol $\left(\mathrm{R}_{1}\right)$ e do catalisador $\left(\mathrm{R}_{2}\right)$. A distância entre o ponto de confluência $\mathbf{y}$ e a entrada da cela de fluxo foi a menor possível (ca. 5 $\mathrm{cm}$ ) visando minimizar perdas de radiação. A altura dos sinais transientes (S) foi empregada como base de medida.

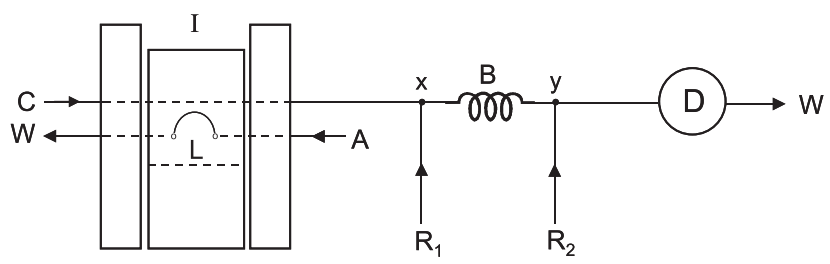

Figura 3. Diagrama de fluxos do módulo de análise empregado para avaliação do desempenho do dispositivo $(D)$. I - injetor; $L$ - alça de amostragem

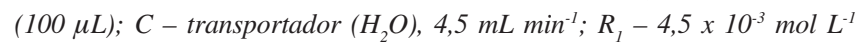

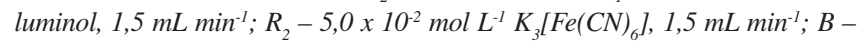
reator helicoidal $(20 \mathrm{~cm}) . W$ - recipiente de descarte

\section{RESULTADOS E DISCUSSÃO}

Apesar da quimiluminescência resultar na emissão de radiação eletromagnética em todas as direções, as configurações usualmente empregadas permitem a medida de apenas uma fração (geralmente $<50 \%$ ) da radiação emitida. Esta limitação resulta em um decréscimo inerente de sensibilidade. $\mathrm{O}$ dispositivo proposto consiste de uma cela de fluxos com reduzida espessura (1 mm) e janela de observação de $78 \mathrm{~mm}^{2}$ (Figura 1). Esta configuração permite maximizar a quantidade de radiação detectada, visto que apenas a radiação emitida no sentido radial não incide sobre os fotodetectores. Adicionalmente, a espessura reduzida permite minimizar absorção de radiação pela solução.

O emprego de dois fotodetectores pode afetar tanto a sensibilidade quanto o nível de ruído. Desta forma, a razão sinal/ruído foi estimada para cada um dos fotodetectores e na configuração que empregava os dois fotodiodos. Para tanto, foram considerados os desvios padrão de 500 leituras da linha base $(1,0-1,7 \mathrm{mV})$ e o sinal máximo referente a uma solução contendo $7,5 \mu \mathrm{mol} \mathrm{L}{ }^{-1} \mathrm{H}_{2} \mathrm{O}_{2}$ (medidas em triplicata). Um aumento significativo da razão sinal/ruído foi observado com o emprego dos dois fotodetectores (Tabela 1), indi-

Tabela 1. Características analíticas do dispositivo proposto empregando 1 ou 2 fotodiodos

\begin{tabular}{cccc}
\hline Fotodiodo & sinal / ruído & Equação da reta & $\mathrm{LD}^{3}\left(\mu \mathrm{mol} \mathrm{L} \mathrm{L}^{-1}\right)$ \\
\hline $\mathrm{a}$ & 28,3 & $\mathrm{~S}=16,8+3,24 \mathrm{C}(\mathrm{r}=0,998)$ & 1,1 \\
$\mathrm{~b}$ & 20,0 & $\mathrm{~S}=26,4+3,27 \mathrm{C}(\mathrm{r}=0,999)$ & 1,6 \\
$\mathrm{a}+\mathrm{b}$ & 54,0 & $\mathrm{~S}=27,5+4,79 \mathrm{C}(\mathrm{r}=0,999)$ & 0,80 \\
\hline
\end{tabular}

1. Estimada considerando os sinais para uma solução $7,5 \mu \mathrm{mol} \mathrm{L} \mathrm{L}^{-1} \mathrm{H}_{2} \mathrm{O}_{2}(\mathrm{n}=3)$ e 500 medidas da linha base; 2. S - sinal máximo (mV); C concentração de $\mathrm{H}_{2} \mathrm{O}_{2}\left(\mu \mathrm{mol} \mathrm{L}{ }^{-1}\right) ; 3$. Limite de detecção estimado a nível de confiança de 99,7\% 
Tabela 2. Comparação de procedimentos para determinação de $\mathrm{H}_{2} \mathrm{O}_{2}$ por quimiluminescência

\begin{tabular}{|c|c|c|c|c|}
\hline Tipo de detector & Geometria da cela & Catalisador & $\left.\mathrm{LD}(\mu \mathrm{mol} \mathrm{L})^{-1}\right)^{\mathrm{a}}$ & Referência \\
\hline fotodiodo & laminar & $\mathrm{K}_{3}[\mathrm{Fe}(\mathrm{CN})]_{6}$ & 0,8 & Presente trabalho \\
\hline fotomultiplicadora & bulbo de vidro & $\mathrm{K}_{3}[\mathrm{Fe}(\mathrm{CN})]_{6}$ & $0,1^{\mathrm{b}}$ & 6 \\
\hline fotomultiplicadora & bulbo de vidro & $\mathrm{K}_{3}[\mathrm{Fe}(\mathrm{CN})]_{6}$ & $0,01^{\mathrm{b}}$ & 7 \\
\hline fotomultiplicadora & espiral de vidro & $\mathrm{Co}(\mathrm{II})$ & 0,01 & 10 \\
\hline fotodiodo & laminar & peroxidase & 0,003 & 11 \\
\hline fotomultiplicadora & - & peroxidase & 8 & 12 \\
\hline
\end{tabular}

a. Limite de deteç̧ão estimado a nível de confiança de 99,7\% (k=3); b. Limite de detecção estimado a nível de confiança de 94,4\% (k=2)

cando que o efeito do aumento da porcentagem de radiação detectada é mais significativo que o aumento no nível de ruído. Outras características analíticas estimadas são mostradas na Tabela 1.

Sinais transientes obtidos com o dispositivo proposto, mostrados na Figura 4, indicam um aumento de sensibilidade de ca. $50 \%$, com o emprego dos dois fotodetectores. Resposta linear foi obtida para concentrações de $\mathrm{H}_{2} \mathrm{O}_{2}$ entre 2,50 e $500 \mu \mathrm{mol} \mathrm{L}{ }^{-1} \mathrm{H}_{2} \mathrm{O}_{2}$ (Tabela 1). Considerando o desvio padrão de 500 leituras da linha base, o limite de detecção foi estimado em $0,8 \mu \mathrm{mol} \mathrm{L}^{-1}$, a nível de confiança de $99,7 \%$. Coeficiente de variação de $0,9 \%$ foi estimado a partir de 20 medidas empregando uma solução contendo $50 \mu \mathrm{mol} \mathrm{L}-1 \mathrm{H}_{2} \mathrm{O}_{2}$.

$\mathrm{O}$ dispositivo proposto apresentou um desempenho analítico comparável ao observado em procedimentos para detecção de $\mathrm{H}_{2} \mathrm{O}_{2}$ em condições químicas similares, ou seja, utilizando hexacianoferrato(III) de potássio como catalisador e detecção com

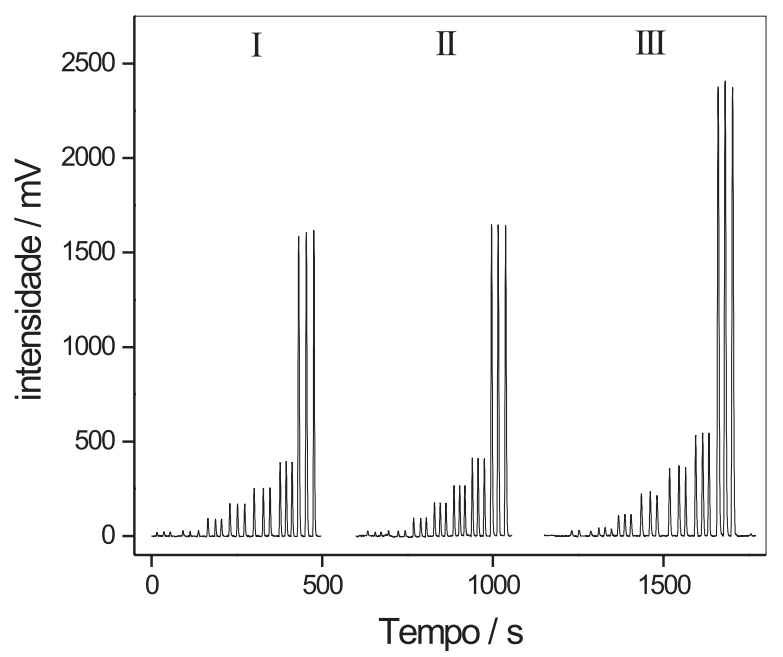

Figura 4. Sinais transientes obtidos com o sistema modelo empregando 1 (I e II) ou 2 fotodetectores (III). Da esquerda para direita, os sinais são referentes a três injeções de 7,5; 10,0; 25,0; 50,0; 75,0; 100,0 e 500,0 $\mu \mathrm{mol}$ $L^{-1}$ de peróxido de hidrogênio fotomultiplicadora $^{6,7}$. Com a utilização do luminol, melhores resultados podem ser alcançados mediante emprego de outros catalisadores e/ou uso de substâncias que prolongam e intensificam a emissão de luz ${ }^{8}$. Uma comparação dos resultados obtidos com o luminômetro proposto com dados da literatura é apresentada na Tabela 2.

$\mathrm{O}$ dispositivo para medidas por quimiluminescência pode ser facilmente construído, com custo estimado em R \$ 450,00 (US\$ 150,00). Foram observadas algumas características favoráveis em comparação com equipamentos comercialmente disponíveis que empregam fotomultiplicadoras, como a maior robustez, menor tamanho e baixo consumo de energia. Estas características são compatíveis com o emprego do instrumento para medidas fora do laboratório como, por exemplo, medidas em campo. Adicionalmente, o dispositivo é bastante atraente para uso em sistemas miniaturizados ou em procedimentos que envolvam o uso de reagentes sólidos ${ }^{9}$, que podem ser diretamente imobilizados na área de observação da cela de fluxo.

\section{AGRADECIMENTOS}

Os autores agradecem às agências FAPESP, CAPES e CNPq pelas bolsas e apoio financeiro.

\section{REFERÊNCIAS}

1. Robards, K.; Worsfold, P. J.; Anal. Chim. Acta 1992, 266, 147.

2. Dodeigne, C.; Lejeune, T. R.; Talanta 1994, 51, 415.

3. Lewis, S. W.; Price, D.; Worsfold, P. J.; J. Biolumin. Chemilumin. 1993, 8, 183.

4. Simpson, M. L.; Sayler, G. S.; Patterson, G.; Nivens, D. E.; Bolton, E. K.; Rochelle, J. M.; Arnott, J. C.; Applegate, B. M.; Ripp, S.; Guillorn, M. A.; Sens. Actuators B 2001, 72, 134.

5. Reis, B. F.; Rocha, F. R. P.; Teixeira, L. S. G.; Costa, A. C. S.; Korn, M.; Quim. Nova 2000, 23, 116.

6. Shaw, F.; Analyst 1980, 105, 11.

7. Bostick, D. T.; Hercules, D. M.; Anal. Chem. 1975, 47, 447.

8. Collaudin, A. B.; Blum, L. J.; Sens. Actuators, B 1997, 88-39, 189.

9. Marshall, R. W.; Gibson, T. D.; Anal. Chim. Acta 1992, 266, 309.

10. Price, D; Mantoura, R. F. C.; Worsfold, P. J.; Anal. Chim. Acta 1998, 377, 145.

11. Hayashi, K.; Sasaki, S.; Ikebukuro, K.; Karube, I.; Anal. Chim. Acta 1998, 329, 127.

12. Li, J.; Wang, K. M.; Yang, X. H.; Xiao, D.; Anal. Commun. 1999, 36,195. 